\title{
Air pollution effects on the intensity of received signal in 3G/4G mobile terminal
}

\author{
Grazia Lo Sciuto ${ }^{1}$ (])
}

Received: 3 November 2018 / Accepted: 20 February 2019 / Published online: 28 February 2019

(c) The Author(s) 2019

\begin{abstract}
In this paper, the existence of the interaction between the atmospheric particulate and the electromagnetic waves attenuation in the frequency band used by the $3 \mathrm{G} / 4 \mathrm{G}$ mobile network was investigated. A few papers present in literature indicate, in presence of air pollution, the possibility of a significant cross polarization of the electromagnetic waves, while none of the papers indicated the possibility of a significant attenuation. This paper has tried to partially fill this gap of knowledge in this field with some new contributions. In all the experiments performed at the department of electrical, electronics and informatics engineering in the University of Catania (87 experiments each one lasting about $36 \mathrm{~min}$ ), significant variations have not been found in the received signal level (RSL), while a polynomial dependency of the RSL local variance from the particulate concentration has been found. To demonstrate that the results obtained in the laboratory have general validity, a campaign test was conducted in an outdoors environment in the frequency band used by the $3 \mathrm{G} / 4 \mathrm{G}$ mobile network. The site of the considered measurement station is selected to represent a typical residential area with high traffic influences in the city of Catania. Also, in this measures campaign conducted in the outdoors environment in the $3 \mathrm{G} / 4 \mathrm{G}$ frequency band, significant variations have not been found in the RSL. While a marked dependence of the RSL local variance from the PM ${ }_{10}$ concentration has been found. This is a noteworthy result, because certain non-negligible RSL local variance variations indicate the possibility of a pronounced cross polarization. The impact of the cross polarization becomes significant for systems reusing frequency, because the cross polarization counteracts the optimum frequency spectrum utilization.
\end{abstract}

Keywords $\mathrm{PM}_{10} \cdot$ Radio signal attenuation $\cdot$ Air pollution

\section{Introduction}

Particles suspended in the atmosphere due to particulate matter emission can have consequences on the electromagnetic wave propagation [1-3]. The electromagnetic wave signal may suffer from attenuation and cross polarization upon encounter with the suspended particles. The main cause of attenuation is the conversion of electromagnetic energy into some other form of energy, such as heat [4].

All theories and techniques for scattering computation are generally based on solving Maxwell's equations either numerically or analytically. Some exact numerical solutions, in turn, often reduce to an analytical solution by means of expanding the incident and scattered fields. Thus, analytical

Grazia Lo Sciuto

glosciuto@dii.unict.it

1 Department of Electrical, Electronics and Informatics Engineering, University of Catania, Catania, Italy and numerical solutions for non-spherical particles are often interwoven. Moreover, in this case, it is impossible to take into account all the factors affecting the solution of a calculus scheme based on Maxwell's equations, so the results obtained will be strongly dependent on the simplifying hypotheses made. For this reason in this paper, to investigate the effect of air pollution on the intensity of received signal in $3 \mathrm{G} / 4 \mathrm{G}$ mobile terminal, two test bed scenarios were considered. In the first test bed scenario, in a controlled indoors environment, the phenomena of the signal attenuation and cross polarization were investigated, while the second test bed scenario, in a outdoors environment, was used to demonstrate that the results obtained in the laboratory have general validity.

In all the experiments performed in a controlled indoors environment (87 experiments each one lasting about 36 min), it significant variations have not been found in the RSL, while a polynomial dependency has been found in the RSL local variance from the particulate concentration. 
Also in the measures campaign conducted in an outdoors environment at $3 \mathrm{G} / 4 \mathrm{G}$ frequency band, significant variations have not been found in the RSL. While a marked dependence of the RSL local variance from the $\mathrm{PM}_{10}$ concentration has been found. This is a noteworthy result because certain non-negligible RSL local variance variations indicate the possibility of a pronounced cross polarization. The impact of cross polarization becomes significant for systems reusing frequency, because the cross polarization counteracts the optimum frequency spectrum utilization.

\section{Materials and methods}

The particulate matter (PM) is defined as a mix of all solid and liquid particles suspended in air. PM can originate from natural processes (soil erosion, forest fires and pollen dispersion) and human activities, typically from combustion processes, road transport and vehicular traffic.

Generally, the particulate consists of solid and liquid particles with a diameter ranging between $100 \mu \mathrm{m}$ and $0.1 \mu \mathrm{m}$. The particles larger than $1 \mu \mathrm{m}$ are generally volatile dust or fly ash, while particles with dimensions smaller than $1 \mu \mathrm{m}$ are called aerosols.

It is possible to distinguish between two important fractions of particulate:

- $\mathrm{PM}_{10}$, consisting of particles with a diameter of less than $10 \mu \mathrm{m}$, these are dangerous since they can penetrate the airways and into the lungs.

- $\mathrm{PM}_{2.5}$, formed by particles of aerodynamic diameter less than $2.5 \mu \mathrm{m}$, are even more dangerous as they can penetrate into the bloodstream.

The $\mathrm{PM}_{10}$ may have both natural and anthropogenic origin. Among the anthropogenic sources, the combustion processes (mainly related to the use of fossil fuels, industrial activities and waste disposal) are the most important. Among the main natural sources, forest fires, volcanic eruptions and pollen dispersal are particularly noteworthy.

Moreover, secondary air pollutants can originate through chemical reactions in the atmosphere such as nitrogen oxides, sulfur dioxide, ammonia and volatile organic compounds, nitrates and ammonium salts [5].

The pollution phenomenon and its entity depend on many factors:

- Source.

- Time of emission and diffusion.

- Time of persistence in the atmosphere.

- Interaction between the pollutant and the air.
Assessing the air pollution by measuring all the compounds introduced into the air is impossible, for this reason to evaluate the extent, it is preferred to detect only some compounds considered indicative to describe the phenomenon [6, 7].

To estimate the attenuation in a communication system, it is necessary to take into account not only the attenuation due to the propagation medium but also all components of which the system is composed [8,9]. In fact, the two mechanisms that can negatively interfere with the frequency spectrum utilization concern the signal attenuation and cross polarization. These phenomena are caused by the dispersion and absorption of the waves in the medium [10]. These two mechanisms are at the base of signal attenuation and strongly depend on the dielectric constant and the particles' size [11].

A few papers indicate, in the presence of air pollution, the possibility of a significant cross polarization of the electromagnetic waves, while none of the papers indicated the possibility of a significant attenuation [10-13]. This paper has tried to partially fill this gap of knowledge in this field with some new contributions. To investigate these phenomena, two test bed scenarios are considered.

In the first test bed scenario, located at the electrotechnical laboratory of the department of electrical, electronics and Informatics engineering in the University of Catania, the experiments consisted of the measurements of the signal intensity generated by the access point Ubiquiti NSM2, which changes the particulate concentration by means of an external particulate source. For the estimates of the effective signal attenuation and particulate matter $\mathrm{PM}_{10}$, measurements of signal strength using the Ubiquiti NanoStation at a frequency of $2.4 \mathrm{GHz}$ are required.

The site of the second test bed scenario is selected to represent a typical residential area with high traffic influences in the city of Catania. The measurement points of the RSL were Gioeni Park and Viale V. Veneto, because these locations have similar orographic conditions and traffic conditions. Both locations are served by the same base station of the $3 \mathrm{G} / 4 \mathrm{G}$ mobile network. The two measuring points are about $2 \mathrm{~km}$ apart. The RSL's measurements were conducted using a smartphone with a dedicated application, called G-Mon. While the $\mathrm{PM}_{10}$ concentration data of the air quality monitoring stations of the municipal city of Catania located at Viale V. Veneto were used. 
The proposed investigation

\section{on the attenuation of radio waves in the presence of atmospheric particulate matter}

The aim of the following experiment is to evaluate if there is an interaction between atmospheric particulate and the attenuation of the signal in a $2.4 \mathrm{GHz}$ radio link and furthermore evaluate how the RSL varies when the concentration of $\mathrm{PM}_{10}$ changes.

\section{Experimental set up: the radio waves generator and the PM analyzer}

The quantitative measurement of the air pollution was carried out using the analyzer AEROCET 531S. Particulate matter is classified according to its size $\left(\mathrm{PM}_{10}, \mathrm{PM}_{7}\right.$, $\mathrm{PM}_{2.5}, \mathrm{PM}_{1}$ ) and as suspended fine dust. Using the analyzer AEROCET, it is possible to determine the density of the particles of the most common dimensions $(0.3 \mu \mathrm{m}, 0.5 \mu \mathrm{m}$, $1.0 \mu \mathrm{m}, 5.0 \mu \mathrm{m}, 10.0 \mu \mathrm{m})$. It also provides the temperature and humidity.

To generate the radio waves, it was used the access point Ubiquiti NSM2. The access point Ubiquiti NSM2 operates in the frequency band centered at $2.4 \mathrm{GHz}$, and makes use of the standard $2 \times 2 \mathrm{MIMO}$ which allows a data transfer speed of $150 \mathrm{Mbit} / \mathrm{s}$, that has an integrated antenna with a maximum amplification of $11.2 \mathrm{~dB}$, and can be used at a temperature not higher than $80^{\circ} \mathrm{C}$ and an humidity not higher than $95 \%$. The intensity of the generated wave by Ubiquiti NSM2 can be variated via the airOS program. The wave intensity default values are represented by different colors of the LEDs.

\section{Experimental set up: the evaluation test system}

The experiment consists of the measurements of the signal intensy generated by the access point Ubiquiti NSM2 in a controlled environment in which the particulate concentration by means of an external particulate source is changed. For these investigations, a rectangular parallelepipedal measurement box of size $200 \times 50 \times 50 \mathrm{~cm}$ has been realized (see Fig. 1).

The structure of this measurement box is realized in wood, and the frame openings are closed by plasticized sheets. The measuring environment was sealed to minimize the influence of uncontrolled particulate sources, such as dust.

The access points Ubiquiti NSM2 and the Aerocet 531s were put internally into the measurement box. The measurement devices were connected to the PC via a network cable. As a source of particulate matter, some incense sticks were also used.

The values of the signal power generated by the Ubiquiti NSM2 access point were collected using the AirOS software, while the concentrations values of the $\mathrm{PM}_{10}$ detected by the Aerocet 531S instrument were collected using the Comet software.

In this paper, three different operating conditions were taken into account:

- Low concentration of $\mathrm{PM}_{10}$.

- Medium concentration of $\mathrm{PM}_{10}$.

- High concentration of $\mathrm{PM}_{10}$.

The first step of all the experiment consists of the measurement of RSL values when no external particulate source is inserted inside the measurement box. The measurement data obtained in this first step must be considered as the

Fig. 1 The measurement box
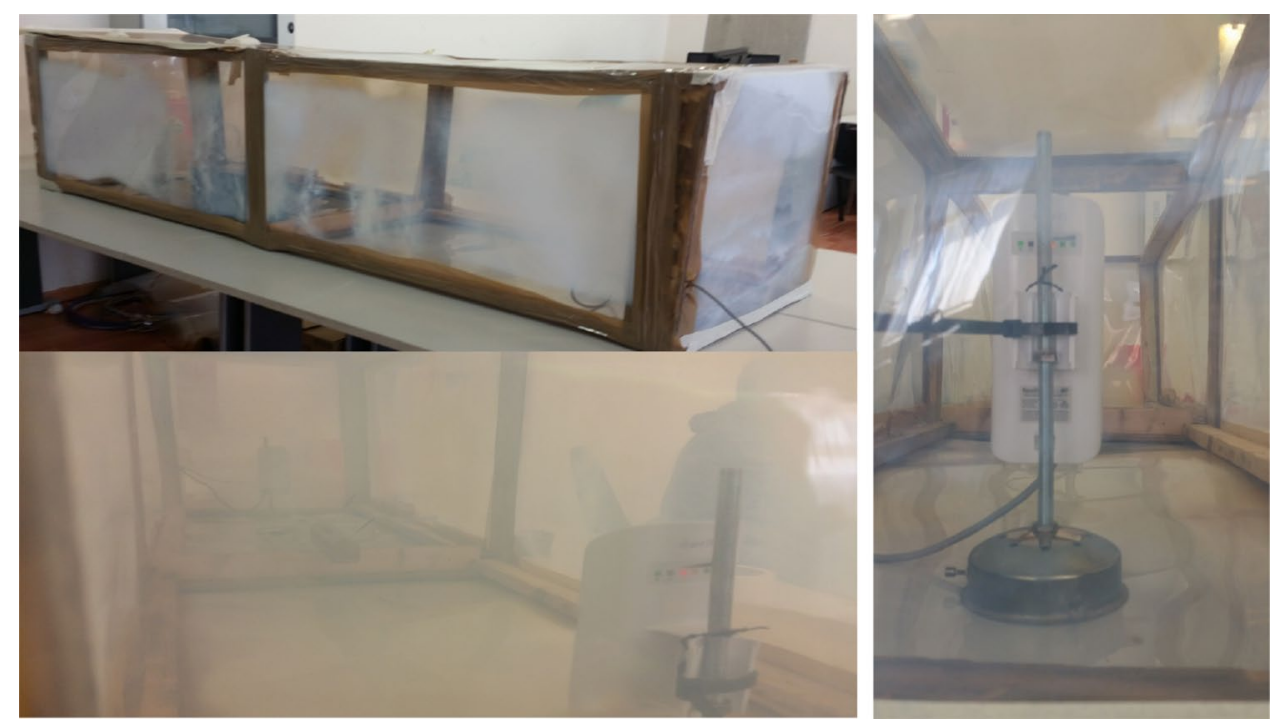
reference. The sampling interval for the RSL is $30 \mathrm{~s}$, while for the $\mathrm{PM}_{10}$ concentrations $60 \mathrm{~s}$.

The second step of the experiments consists of changing the concentration of $\mathrm{PM}_{10}$ by inserting inside the structure a burning incense stick and measuring the RSL every $30 \mathrm{~s}$. Also in this step, the measurements' sampling interval of the $\mathrm{PM}_{10}$ concentrations is $60 \mathrm{~s}$.

The third step of the experiments consists of using more particulate sources to obtain a very high concentration of $\mathrm{PM}_{10}$ inside the measurement box.

\section{The proposed investigation on the attenuation of radio waves in an outdoors environment}

To demonstrate that the results obtained in the laboratory have general validity, a campaign test was conducted in an outdoors environment in the frequency band used by the 3G/4G mobile network. The site of the considered measurement station is selected to represent a typical residential area with high traffic influences in the city of Catania.

Catania is an urban area with a high density of habitants and has the same air pollution problems as other big cities in the world. These problems are getting worse due to bad city planning and the city's topographical features. However, the geography of this city aids the dispersion of air pollutants. The main air pollutant emissions is from transport vehicles and industrial activities.

Catania is one of the metropolitan cities in Italy that is densely populated, with air pollution attributed to the effects of Airport International Catania and high motorization; its population is declared as over 1885.9 inhabitants per square kilometer. Many interesting factors play a fundamental role in the variability of air flow: morphological and topographical conditions, the influence of volcanic passive degassing on the air quality of Mt. Etna, as well as the development of sea and land breeze circulation systems. The population of Catania and its vehicular traffic are concentrated within the city. In nearly every case, urban congestion translates into traffic congestion. Most of the people have automobiles (65,000 vehicles), motorcycles, and motor scooters. But in addition to this routine urban transportation, for instance, the vehicular pollution is considered to be the most significant source of urban pollution as well as the main source of $\mathrm{PM}_{10}$, which is not significantly related to the very limited industrial emissions. The network of air quality monitoring stations in Catania was designed and has been managed by Direzione Ecologia e Ambiente since 1992.

In this study, the concentrations of air pollution of the different monitoring stations located in Catania are used (see Fig. 2). In fact, the municipal city of Catania provided four monitoring stations (see Fig. 3) to measure $\mathrm{PM}_{10}, \mathrm{NO}_{2}, \mathrm{NO}$, $\mathrm{NO}_{X}$ concentrations.

The $\mathrm{PM}_{10}$ concentration data were last collected by the Direzione Ecologia e Ambiente P.O. Prevenzione Inquinamento of the municipal city of Catania during the year 2012. The concentrations were collected using a data storage device and electronic spreadsheet handled the data in periods of $24 \mathrm{~h}$, every day, in agreement with norms established by Directive 2008/50/EC of the European Parliament and of the Council on ambient air quality and cleaner air for Europe. The data set of 864 recorded concentrations contained missing values due to instrument malfunction and calibrations.

The measurement points of the RSL were Gioeni Park and Viale V. Veneto, because these locations have similar orographic conditions and traffic conditions. Both locations are served by the same base station of the $3 \mathrm{G} / 4 \mathrm{G}$ Mobile
Fig. 2 Map of the city of Catania, Sicilia showing the location of the stations for pollutant concentration

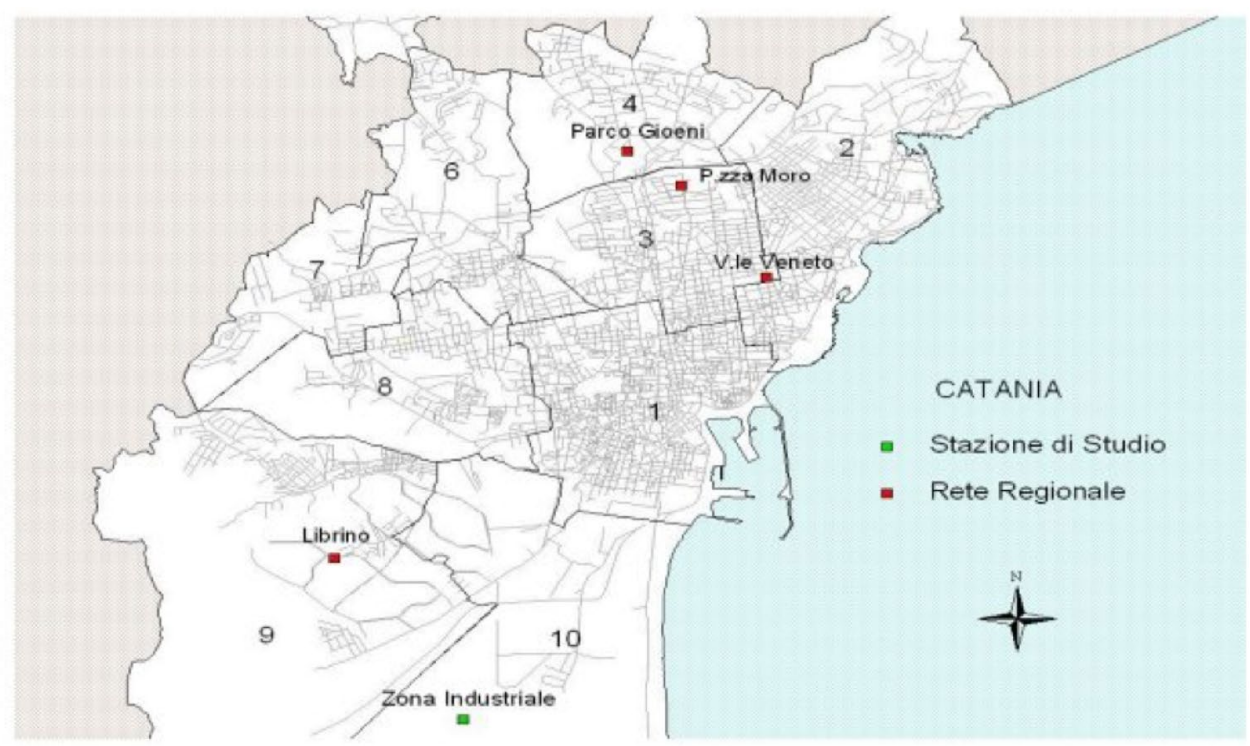




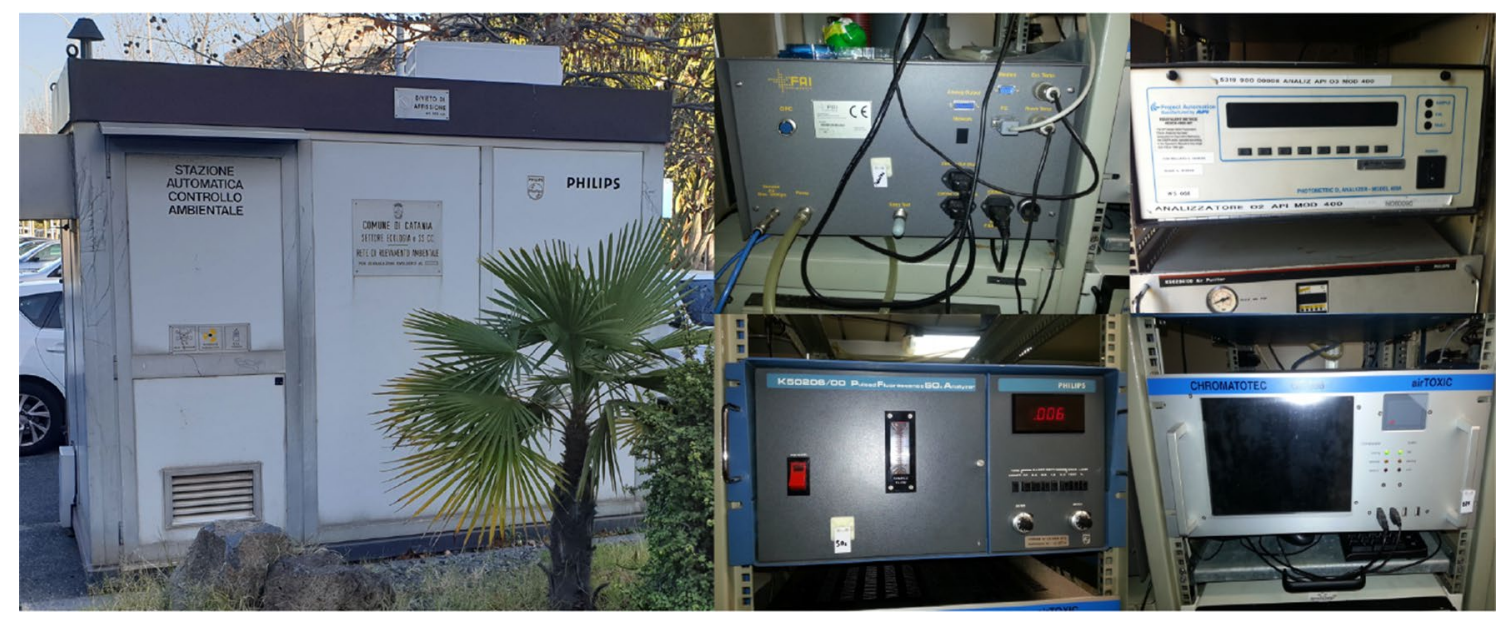

Fig. 3 A monitoring station with some of its equipments

network. The two measuring points are about $2 \mathrm{~km}$ apart. While the $\mathrm{PM}_{10}$ concentration data of the monitoring station located at Viale V. Veneto were used.

The RSL's measurements were conducted using a smartphone with a dedicated application, called G-Mon, able to export in CVS format a complete report during time regarding several network and signal parameters such as the received signal strength indicator (RSSI), the reference signal received power (RSRP), the reference signal received quality (RSRQ), the signal-to-noise ratio (SNR), the location area code (LAC), the cell ID (CID), and the connection type (LTE, HSPA, UMTS, 4G, etc.). More specifically, we analyzed the RSSI to evaluate the attenuation and the signal fluctuation. The knowledge of the cell ID is very important, because it allows the verification of the measured RSL if it refers to the same base station. The application allows the collection of data with a programmable frequency. For this experiment, the data were recorded at a frequency of $60 \mathrm{~s}$.

\section{Results and discussion}

\section{Indoors environment}

In Fig. 4, the signal waveform with respect to time in absence of particulate (a), the signal waveform with respect to the variation of the particulate concentration (b), and the variation of the particulate concentration versus time are shown.

The maximum number of particles for $\mathrm{m}^{3}$ that the instrument can detect is $13,000 \mu \mathrm{g} / \mathrm{m}^{3}$. For this reason, the data collected in the third phase of the experience were not considered in the analysis, because the concentration of particulates exceeds the value of $13,000 \mu \mathrm{g} / \mathrm{m}^{3}$.
To better analyze the variability of the signal in the presence of particulates, a statistical study was performed on the RSL local variance using a mobile window with amplitude of four samples.

Figure 5 shows that when the particulate sources are introduced (instant indicated by the red line in the figure), the RSL variance, calculated through the sliding window, reaches much higher values.

The red line depicted in the graphs indicates the time in which the $\mathrm{PM}_{10}$ sources were inserted. As can be observed from Fig. 4 , in absence of the particulate the signal level ranged from -26 to $-20 \mathrm{dBm}$ and that even when the $\mathrm{PM}_{10}$ sources were inserted there were not significant variations in the RSL. Moreover, as shown in Fig. 5, when the external sources of $\mathrm{PM}_{10}$ are inserted, the signal has a significant increase in its value of local variance, in fact before the insertion of the $\mathrm{PM}_{10}$ sources, the local variance ranged from 0 to 37, while after the insertion of the $\mathrm{PM}_{10}$ sources, the local variance ranged from 0 to 114 . Finally, there were no appreciable variations in the value of average signal level.

In all the performed experiments (87 experiments each one lasting about $36 \mathrm{~min}$ ), significant variations have not been found in the RSL, while a polynomial dependency of the RSL local variance from the particulate concentration has been found.

$\operatorname{var}(\mathrm{RSL})=p_{1} \times\left(\mathrm{PM}_{10}\right)^{2}+p_{2} \times\left(\mathrm{PM}_{10}\right)+p_{3}$,

where the parameters values $p_{1}, p_{2}, p_{3}$ of Eq. (1) calculated using the data of all the 87 experiments in the least-squares sense are $-0.000000247,0.011,10.35$, respectively.

The results revealed that no significant attenuation effects occur in RSL in the presence of particulate matter. On the other hand, significant variations in the mean signal level (local variance) make very likely the possibility of more significant cross polarization. The impact of 
Fig. 4 a Signal level obtained in case of no incense sticks inside the measurement box. b Signal level obtained in the presence of incense sticks inside the measurement box. c Variation of the particulate concentration versus time
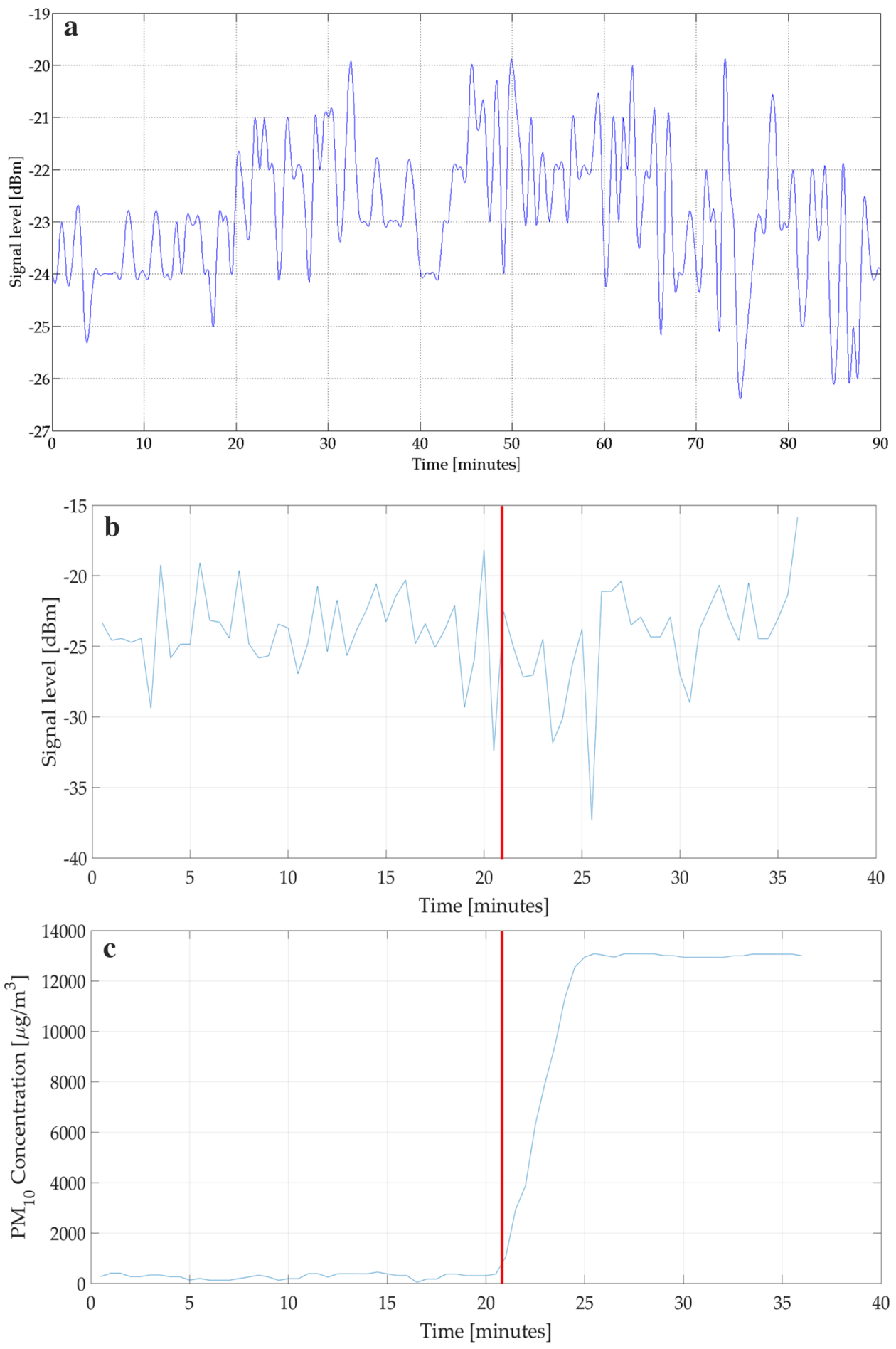

cross polarization becomes significant for systems reusing frequency, because the cross polarization counteracts the optimum frequency spectrum utilization.

Figure 6 shows that the model for local variance, obtained in this paper, is in good agreement with the experimental data.

\section{Outdoors environment}

Also in this measures campaign conducted in an outdoors environment at $3 \mathrm{G} / 4 \mathrm{G}$ frequency band, significant variations have not been found in the RSL. While the 
experimental data show a marked dependence of the RSL local variance from the $\mathrm{PM}_{10}$ concentration.

Although the $\mathrm{PM}_{10}$ concentration values measured by the monitoring station are significantly lower than those obtained in the laboratory (see Fig. 7), the polynomial dependency of the RSL local variance from the particulate concentration holds. In this case, the parameters values
Fig. 5 Signal level variance of signal in the presence of incense sticks inside the measurement box

Fig. 6 Dependency of the RSL local variance from the particulate concentration: experimental data and model data obtained by the Eq. (1)

Fig. 7 The air pollutant concentrations in a typical spring work day measured by the monitoring station located at Viale V. Veneto
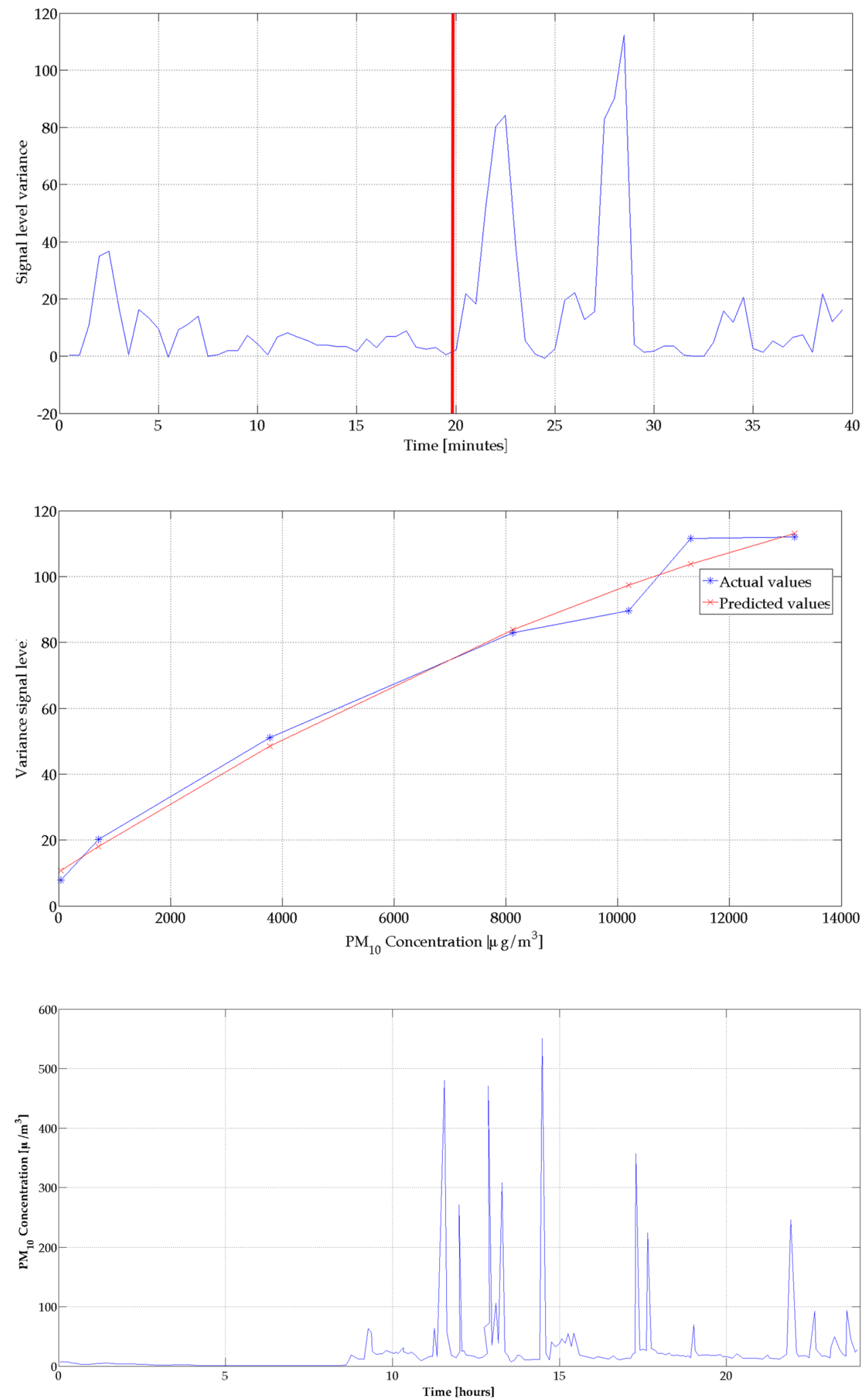
$p_{1}, p_{2}, p_{3}$ of Eq. (1) are $-0.00030552,0.4615,8.740$, respectively.

Figure 8 shows the RSL local variance in a typical spring work day measured by the monitoring station located at Viale V. Veneto.

As shown in Fig. 9, also in this case, the formula for the RSL local variance, obtained in this paper, is in good agreement with the experimental data. This demonstrates that the results obtained in the laboratory have general validity.

\section{Conclusion}

The analysis carried out in this paper was aimed to investigate the existence of an interaction between the $\mathrm{PM}_{10}$ concentration and the propagation of electromagnetic waves. For these investigations, a rectangular parallelepipedal measurement box in wood of size $200 \times 50 \times 50 \mathrm{~cm}$ was realized to perform the measurements in a controlled environment. The access points were put internally into the measurement box and connected to the PC via a network cable. The Ubiquiti antennas were used to transmit a signal at $2.4 \mathrm{Ghz}$ frequency, and the Aerocet 531S was used to detect the concentration of the particulates. The data were collected in two different conditions: with and without an external particulate source.

In all the performed experiments (87 experiments each one lasting about $36 \mathrm{~min}$ ), significant variations have not been found in the RSL, while a polynomial dependency of the RSL local variance from the particulate concentration has been found.

To demonstrate that the results obtained in the laboratory have general validity, a campaign test was conducted in an outdoors environment in the frequency band used by the 3G/4G mobile network. The site of the considered measurement station was selected to represent a typical residential area with high traffic influences in the city of Catania. Also in this measures campaign conducted in an outdoors environment in the $3 \mathrm{G} / 4 \mathrm{G}$ frequency band significant variations in the RSL have not been found. While a marked dependence of the RSL local variance from the $\mathrm{PM}_{10}$ concentration has been found. This is a noteworthy result because certain non-negligible RSL local variance variations indicate the possibility of a pronounced cross polarization. The impact of cross polarization becomes significant for systems reusing
Fig. 8 Signal level variance obtained by processing the RLS data measured in Parco Gioeni on the same day of those concerning the $\mathrm{PM}_{10}$ concentrations reported Fig. 7
Fig. 9 Dependency of the RSL local variance from the particulate concentration in an outdoors environment: experimental data and model data obtained by the Eq. (1)
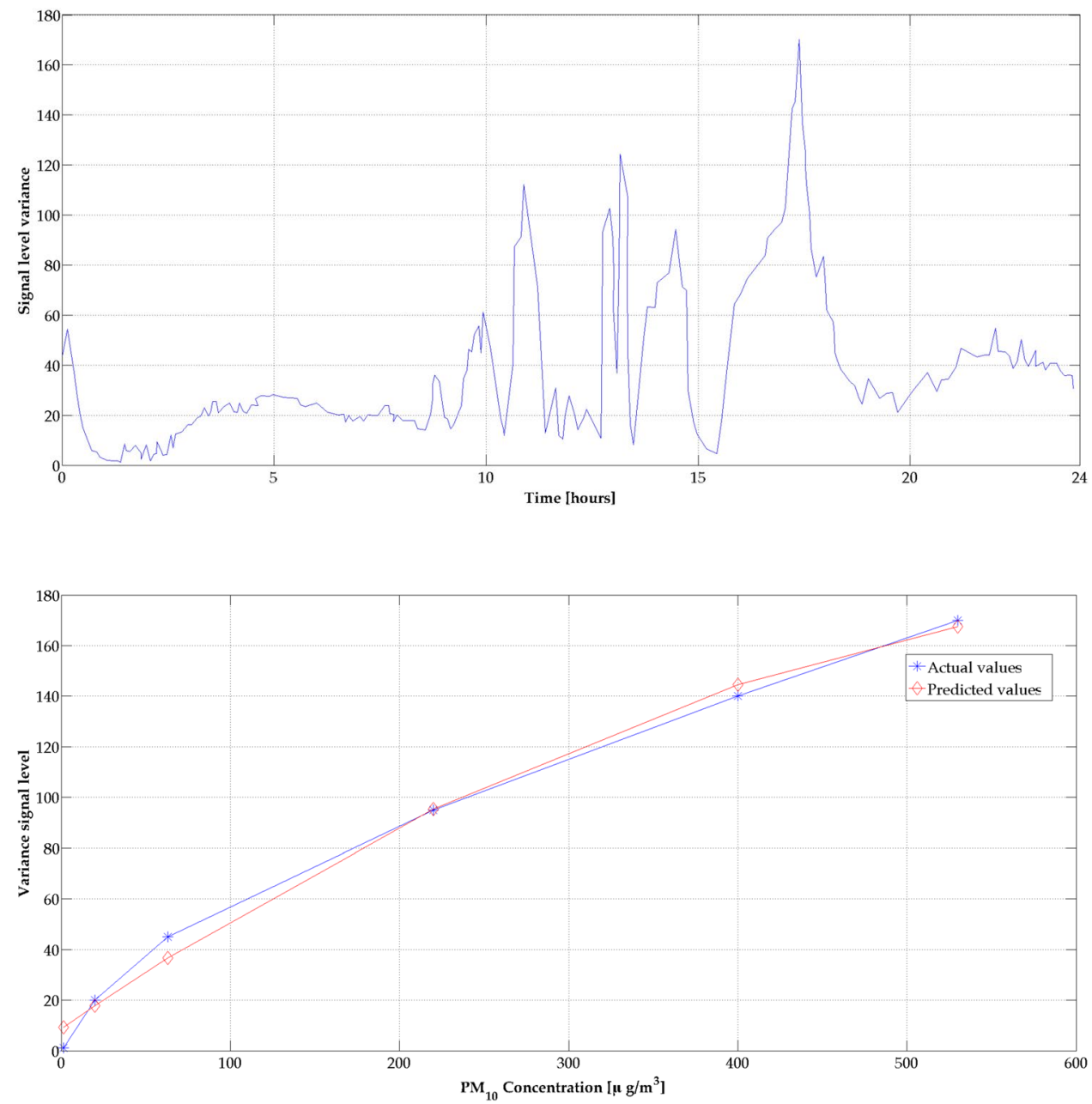
frequency, because the cross polarization counteracts the optimum frequency spectrum utilization.

Acknowledgements The author wish to thank the Department of Electrical, Electronics and Computer Engineering of the University of Catania for research facilities and support provided within the framework of Departmental Research Program 2016/2018.

\section{Compliance with ethical standards}

Conflict of interest On behalf of all authors, the corresponding author states that there is no conflict of interest.

Open Access This article is distributed under the terms of the Creative Commons Attribution 4.0 International License (http://creativeco mmons.org/licenses/by/4.0/), which permits unrestricted use, distribution, and reproduction in any medium, provided you give appropriate credit to the original author(s) and the source, provide a link to the Creative Commons license, and indicate if changes were made.

\section{References}

1. Lau, L., He, J.: Investigation into the effect of atmospheric particulate matter (pm2. 5 and pm10) concentrations on gps signals. Sensors 17(3), 508 (2017)

2. David, N., Gao, H.O.: Using cellular communication networks to detect air pollution. Environ. Scie. Technol. 50(17), 9442-9451 (2016)

3. Paun, M., Marghescu, I., Tamas, R.: A software radio approach for locating unintentional ozone-generating sources. In: 2015 IEEE International Black Sea Conference on Communications and Networking (BlackSeaCom), pp. 107-111. IEEE (2015)

4. Shang, P., Shen, F.: Atmospheric correction of satellite gf-1/wfv imagery and quantitative estimation of suspended particulate matter in the yangtze estuary. Sensors 16(12), 1997 (2016)

5. Brusca, S., Famoso, F., Lanzafame, R., Mauro, S., Messina, M., Strano, S.: Pm10 dispersion modeling by means of cfd $3 \mathrm{~d}$ and
Eulerian-Lagrangian models: analysis and comparison with experiments. Energy Procedia 101, 329-336 (2016)

6. García-Sánchez, C., Van Tendeloo, G., Gorlé, C.: Quantifying inflow uncertainties in RANS simulations of urban pollutant dispersion. Atmos. Environ. 161, 263-273 (2017)

7. Zhu, S., Lian, X., Wei, L., Che, J., Shen, X., Yang, L., Qiu, X., Liu, X., Gao, W., Ren, X., et al.: Pm2. 5 forecasting using SVR with PSOGSA algorithm based on CEEMD, GRNN and GCA considering meteorological factors. Atmos. Environ. 183, 20-32 (2018)

8. Solheim, F.S., Vivekanandan, J., Ware, R.H., Rocken, C.: Propagation delays induced in GPS signals by dry air, water vapor, hydrometeors, and other particulates. J. Geophys. Res. Atmos. 104(D8), 9663-9670 (1999)

9. Beritelli, F., Capizzi, G., Sciuto, G.L., Napoli, C., Scaglione, F.: Rainfall estimation based on the intensity of the received signal in a LTE/4G mobile terminal by using a probabilistic neural network. IEEE Access. 6, 30865-30873 (2018)

10. Harb, K., Butt, O., Al-Yami, A.A., Abdul-Jauwad, S.: Probabilistic dust storm layers impacting satellite communications. In: 2013 IEEE International Conference on Space Science and Communication (IconSpace), pp. 407-411. IEEE (2013)

11. Musa, A., Bashir, S.O., Abdalla, A.H.: Review and assessment of electromagnetic wave propagation in sand and dust storms at microwave and millimeter wave bands-part II. Progr. Electromagn. Res. 40, 101-110 (2014)

12. Musa, A., Paul, B.: Prediction of electromagnetic wave attenuation in dust storms using MIE scattering. In: AFRICON, 2017 IEEE, pp. 603-608. IEEE (2017)

13. Chinthaka, M.D., Malka, N.H., Ramamohanarao, K., Moran, B., Farrell, P.: The signal propagation effects on IEEE 802.15. 4 radio link in fire environment. In: 2010 5th International Conference on Information and Automation for Sustainability (ICIAFs), pp. 411-414. IEEE (2010)

Publisher's Note Springer Nature remains neutral with regard to jurisdictional claims in published maps and institutional affiliations. 